\title{
Bronchiolitis obliterans organizing pneumonitis primed by adjuvant radiotherapy in breast cancer patient in comparison with COVID-19 pneumonia: a
}

\section{case series}

\begin{abstract}
Adjuvant radiotherapy (RT) is a milestone in breast cancer conserving treatment. The lung and heart are the main organs at risk to be considered when an external beam RT is planned regardless of the chosen modality (3D conformal or IMRT). Although many efforts to comply with organ at risk constraints and minimize dose to them are observed in planning, unexpected severe toxicities could occur like pneumonitis outside the radiation port due to Bronchiolitis Obliterans Organizing Pneumonitis (BOOP) primed by radiotherapy. Its clinical and radiological diagnosis do not meet criteria of a classical radiation pneumonitis but findings seem to be similar to COVID-19 (CO-19) pneumonia. At the COVID-19 pandemic time as at present, these clinical and CT findings similarities could yield confounding interpretations in diagnosis and lead to unnecessary panic induced scenarios with an admission of the suspected affected patient in isolation pathway. Moreover CO-19 pneumonia diagnosis may be complicated when negative swab and false negative RealTime Polymerase Chain Reaction (RT-PCR) are recorded. Broncholavage fluid (BAL) and specific serology together to adequate anamnesis may help clinicians in making a correct diagnosis and report. Herein a case series of two case reports showing many overlapping features between RT-BOOP and CO-19 pneumonia in two patients with negative swab and RT-PCR CO-19 test.
\end{abstract}

Keywords: radiation ports, RT-PCR, BAL, ground glass opacities
Volume II Issue 4 - 2020

\author{
Grazia Lazzari,', Caterina Malcangi,', Elda \\ Chiara Resta, ${ }^{2}$ Ernesto D'Ettorre, ${ }^{2}$ Giovanni \\ Silvano' \\ 'Radiation Oncologist, Radiation Oncology Unit, San Giuseppe \\ Moscati Hospital, Italy \\ ${ }^{2}$ Radiologist, Radiology-Covid Unit, San Giuseppe Moscati \\ Hospital, Italy
}

Correspondence: Grazia Lazzari, Radiation Oncologist, Radiation Oncology Unit, San Giuseppe Moscati Hospital, Road to Martina Franca, 74I00 Taranto, Italy, Tel +39 0994585 I80, Email lazzarigrazia@gmail.com

Received: July 0I, 2020 | Published: July 16, 2020

\section{Introduction}

RT-BOOP syndrome is a rare atypical event due to a migratory pneumonitis showing clinical features of a flu-like syndrome and Computed Tomography (CT) findings of nodular involvement in both lungs but outside the radiation fields. ${ }^{1}$ This event has an incidence of $1.8-2.9 \%$, and is typical of irradiated breast cancer occurring after several month later adjuvant RT. ${ }^{2} \mathrm{CT}$ images show a wide pictorial fashion of features getting from focal nodular or mass-like opacities to areas of consolidation resembling pneumonia or patchy ground glass infiltrates with an air bronchogram or vessel sign as characteristic pictures. ${ }^{3}$ In the CO-19 pandemic time much clamour has occurred in light of the published evidences on clinical and radiological findings about CO-19 pneumonia. ${ }^{4}$ In this novel disease, chest CT scan has been considered helpful to early recognition and monitoring CO-19 patients especially in those with false negative RT-PCR with or without symptoms. Typical and atypical CT pictures have been published with the aim to help familiarize radiologists with these features and make a correct diagnosis. ${ }^{5}$ The similarity of clinical and radiological signs between these two diseases may mislead either radiologists and clinicians to make incorrect reports in CO -19 swab and RT-PCR false negative carriers while are waiting for BAL results and serology.

\section{Case reports}

Informed consent to data treatment was obtained by each patient. The Hospital Ethical Committee approved the publication of these case reports.

\section{Case No I}

This patient was a 50 years old woman with an invasive ductal G2 carcinoma of the right breast staged pT2 pN0 M0 with Luminal A phenotype. Anamnesis was negative for diabetes, opportunistic infections, autoimmune diseases. After conservative surgery, she had Tamoxifen and underwent adjuvant RT with 3dimensional conformal technique and conventional fractionation $(2 \mathrm{~Gy} / 50 \mathrm{~Gy})$ plus a $10 \mathrm{~Gy}$ boost on the tumor bed. Radiation treatment plan consisted of two Multi leaf Collimator customized $6 \mathrm{MV}$ tangential fields depicting a typical dose distribution around the right breast. The right lung V20 Gy was $<20 \%$ and MLD was $<8$ Gy. During the treatment, no acute toxicity was recorded; the patient completed the RT treatment at the end of January 2020. Two months later, the patient returned to our observation complaining of a flu-like syndrome, cough, fever, moderate-severe dyspnea due to an acute pneumonitis. The CT scan images showed in the upper right lobe some areas of consolidation resembling pneumonia and in both lungs many ground -glass opacities with the bronchus sign (Figure 1). Swab and RT-PCR were negative for CO-19; the specific serology and BAL confirmed the absence of the virus infection. BAL consisted of inflammatory cells showing many neutrophilis and activated lymphocytes. The virus was not detected after steroid assumption, the symptoms drastically ameliorated. The $\mathrm{CT}$ scan showed a complete resolution of the previous findings in both lungs; (Figure 2). The final diagnosis was BOOP.

\section{Case No 2}

This 45 years old woman, in the beginning of March 2020, presented with a febrile flu-like syndrome with cough and mild 
dyspnea. High Erythrocyte Sedimentation Rate (ESR) and leukocytosis with low lymphocyte count were found. D-dimer level was low. The CT scan images showed in both lungs several nodular opacities resembling pneumonia associated to a vessel sign and air bronchogram (Figure 3). Swab and RT-PCR were negative for CO-19 infection but BAL and serology confirmed the virus as the responsible agent. Patient was admitted in a CO-19 Unit in anisolated and dedicated centre and received specific symptomatic therapy. The pneumonia ameliorated. Chest CT scan images showed a complete resolution of nodular resembling pneumonia (Figure 4). Multinodular COVID-19 pneumonia was the final diagnosis.

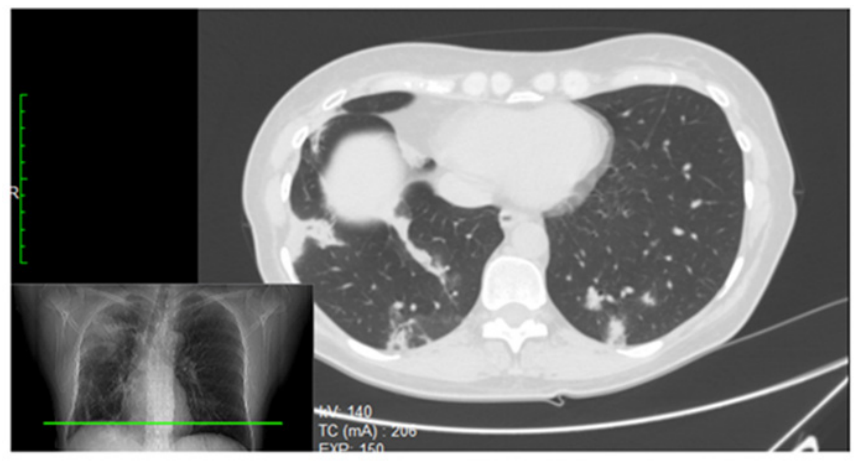

Figure I CT scan of the chest at baseline.

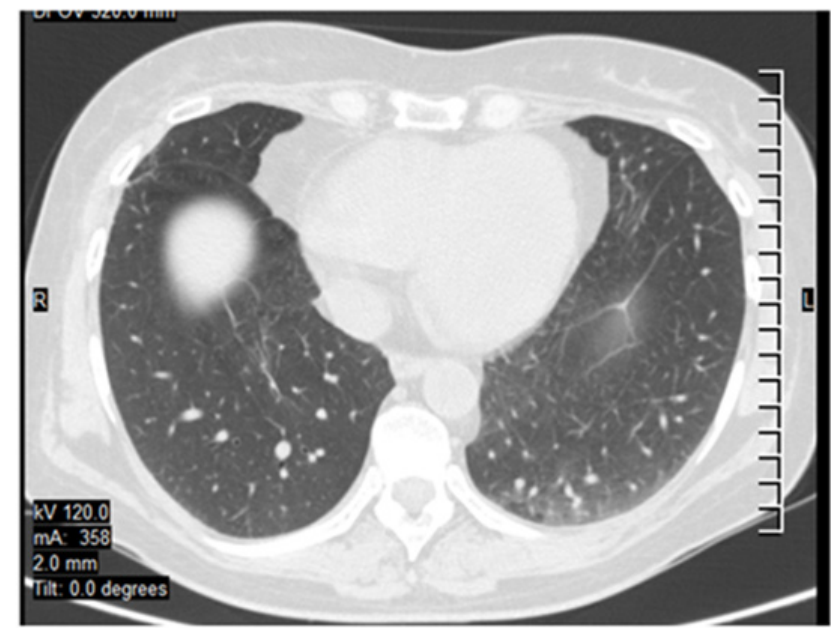

Figure 2 CT scan of the chest after steroid therapy.

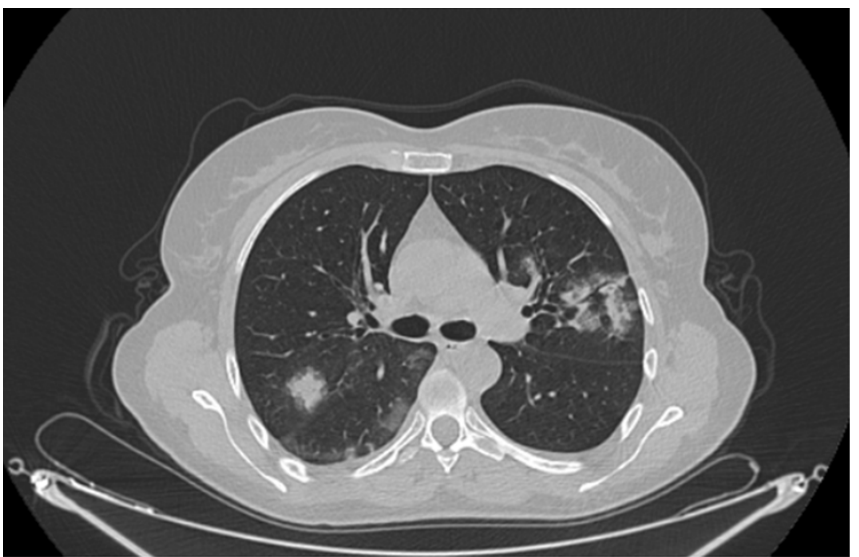

Figure 3 CT scan of the chest at diagnosis of Covid 19-pneumonia.

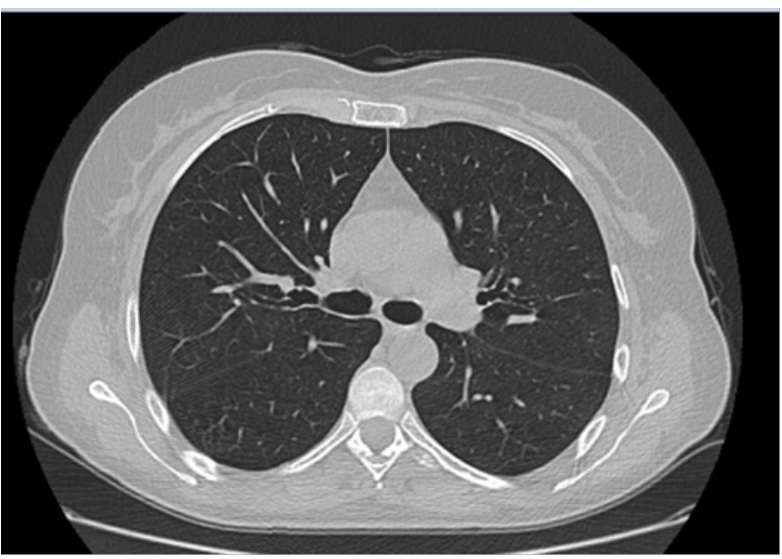

Figure 4 CT scan after therapy.

\section{Discussion}

Radiation pneumonitis (RP) is a common side effect of thoracic RT. ${ }^{6}$ As outlined by Ippolito et al. ${ }^{7}$ findings of classic RP are usually unilateral, involving the ipsilateral lung under the irradiated breast. Further the distribution of chest CT abnormalities, like nodular opacities or ground glass opacities, correspond to the radiation treatment fields through the radiation ports. However atypical pneumonitis may be primed by RT as the so called "Bronchiolitis Obliterans Organizing Pneumonitis" (RT-BOOP), which show clinical and CT findings different from classic radiation pneumonitis but similar to CO-19 pneumonia. In RT-BOOP syndrome, there is a migratory pneumonitis whose radiological findings are commonly seen outside the radiation fields with a bilateral lung involvement. ${ }^{1}$ Like a febrile flu-like syndrome, it has been observed several months after adjuvant RT in breast cancer patients with an incidence of 1.8$2.9 \%$. Tamoxifen, smoke and age have been related too. ${ }^{8}$ From all the reported series, a spectrum of CT findings in RT-BOOP have been described getting from focal nodular or mass-like opacities to areas of consolidation resembling pneumonia or patchy ground glass infiltrates. Interestingly, in nodular opacities, either a feeding vessel or bronchus sign like an air bronchogram have been identified as characteristic signs. ${ }^{3}$ These syndromes have a good prognosis and are sensitive to steroid therapy, developing a migratory pattern with relapse over time while tapering or after stopping steroid therapy. ${ }^{9}$ When performed, BAL shows a mixed cellular pattern with increased lymphocytes, neutrophils, and eosinophils; no trace of virus or opportunistic are found. ${ }^{10}$ Although primed by RT, the related mechanism is unknown; it has been hypothesized a hypersensitivity phenomenon due to genetically susceptible host and/or an as-yet unrecognized triggering factor acting on "radiation-primed" lymphocytes to produce a pneumonitis. ${ }^{11}$

CO-19 pneumonia is the main event described in CO-19 pandemic time, occurring as a febrile flu-like syndrome with cough and dyspnea in the majority of the described cases or evolving into dramatic and fatal complications in several cases. ${ }^{4}$ Many studies have assessed the key role of CT in diagnosis of CO-19 patients mainly with false negative RT-PCR results, showing a $98 \%$ sensitivity in detecting and monitoring the course of the pneumonia. ${ }^{5}$ Radiologists have described the hallmark of CT findings with some hints on the temporal changes over the course of this sometimes life-threatening disease, reviewing the typical and atypical CT findings in this novel disease. As a result, the CT hallmarks of COVID-19 have been described as bilateral 
distribution of ground glass opacities with or without consolidation in the posterior and peripheral lung. The predominant findings in later phases include consolidations, linear opacities, "crazy-paving" pattern, "reversed halo" sign and vascular enlargement. ${ }^{12}$ Thus, how to discern between these two similar but different rare diseases? In our experience, the anamnesis of a recent breast RT together with the negativity of CO-19 tests and BAL fluid were helpful to exclude the viral infection.

\section{Acknowledgments}

None.

\section{Conflicts of interest}

The authors declare no conflicts of interest.

\section{Funding}

None.

\section{References}

1. Crestani B, Valeyre D, Roden S, et al. Bronchiolitis obliterans organizing pneumonia syndrome primed by radiation therapy to the breast. $\mathrm{Am} J$ Respir Crit Care Med. 1998;158(6):1929-1935.

2. Oie Y, Saito Y, Kato M, et al. Relationship between radiation pneumonitis and organizing pneumonia after radiotherapy for breast cancer. Radiother Oncol. 2013;8:56.

3. Kubo A, Osaki K, Kawanaka T, et al. Risk factors for radiation pneumonitis caused by whole breast irradiation following breast-conserving surgery. $J$ Med Invest. 2009;56(3-4):99-110.
4. Kakodkar P, Kaka N, Baig MN. A Comprehensive Literature Review on the Clinical Presentation, and Management of the Pandemic Coronavirus Disease 2019 (COVID-19). Cureus. 2020;12(4):e7560.

5. Huang P, Liu T, Huang L, et al. Use of chest CT in combination with negative RT-PCR assay for the 2019 novel coronavirus but high clinical suspicion. Radiology. 2020:295(1):22-23.

6. Choi YW, Munden RF, Erasmus JJ, et al. Effects of radiation therapy on the lung: radiologic appearances and differential diagnosis. Radiographics. 2004;24(4):985-997.

7. Ippolito E, Fiore M, Greco C, et al. COVID-19 and radiation induced pneumonitis: Overlapping clinical features of different diseases. Radiother Oncol. 2020;148:201-202.

8. Murofushi KN, Oguchi M, Gosho M, et al. Radiation-induced bronchiolitis obliterans organizing pneumonia (BOOP) syndrome in breast cancer patients is associated with age. Radiat Oncol. 2015;10:103.

9. Ogo E, Komaki R, Abe T, et al. The clinical characteristics and nonsteroidal treatment for radiation-induced bronchiolitis obliterans organizing pneumonia syndrome after breast-conserving therapy. Radiother Oncol. 2010;97:95-100.

10. Allen JN, Davis WB, Pacht ER. Diagnostic significance of increased bronchoalveolar lavage fluid eosinophils. Am Rev Respir Dis. 1990;142(3):642-647.

11. Roberts CM, Foulcher E, Zaunders JJ, et al. Radiation pneumonitis: a possible lymphocyte-mediated hypersensitivity reaction. Ann Intern Med. 1993;118(9):696-700.

12. Carotti M, Salaffi F, Sarzi-Puttini P, et al. Chest CT features of coronavirus disease 2019 (COVID-19) pneumonia: key points for radiologists. Radiol Med. 2020;125(7):636-646. 